SECTION 2. Applied mathematics. Mathematical modeling.

Shevtsov Alexandr Nikolayevich candidate of technical Sciences, President of International Academy of Theoretical \& Applied Sciences, Kazakhstan $\underline{\text { Shev_AlexXXXX@mail.ru }}$

\title{
SOME QUESTIONS SIMULATION OF INTERACTIVE DYNAMIC SYSTEMS
}

Abstract: The process of developing an interactive model is a rather complicated process. In the structure of the model is necessary not only to draw up the conditions that influence its work, and also to connect directly with the graphic libraries and the matrices of transformation of the coordinates. In this article the approach and the implementation of the three-dimensional model calculation of the orbital trajectories of objects interactively in the Delphi-XE4.

Key words: interactive model, orbits, collisions of objects.

\section{НЕКОТОРЫЕ ВОПРОСЫ МОДЕЛИРОВАНИЯ ИНТЕРАКТИВНЫХ ДИНАМИЧЕСКИХ СИСТЕМ}

Аннотация: Процесс реализации любой интерактивной модели представляет собой довольно сложный процесс. В самой структуре модели необходимо не только правильно составить условия, влияющие на ее работу, но и связать непосредственно с графическими библиотеками и матрицами преобразования координат. B данной статье рассмотрен подход, и реализация трехмерной модели расчета орбитальных траекторий объектов, в интерактивном режиме в среде Delphi-XE4.

Ключевые слова: интерактивная модель, орбиты, столкновения объектов.

Отслеживание траекторий движения объектов на орбите Земли представляет собой очень важную проблему. По данным ежеквартального отчета НАСА, посвященного проблеме космического мусора, на данный момент на орбите находятся 16,094 тысячи "мусорных объектов", в том числе вышедших из строя 3,396 тысячи спутников и 12,968 тысячи верхних ступеней ракет-носителей и других обломков. К примеру, ВВС США готовят к запуску первый космический аппарат, предназначенный для наблюдения за космическим мусором на орбите Земли. Спутник космического наблюдения будет следить за 1000 действующими спутниками и 22000 фрагментами космического мусора. Три компании, в том числе, Lockheed-Martin, получили от ВВС США по 30 миллионов долларов на разработку электронной изгороди, которая позволит отслеживать перемещения космических объектов размером более пяти сантиметров [1].

Причем большинство объектов не являются геостационарными, а значит движутся по определенным эллиптическим орбитам. Зная орбитальные координаты объектов, можно проанализировать данные и отследить движение всех объектов. Разработаем математическую и компьютерную модель, движения объектов на орбите Земли. Из курса физики известно, что тело, движущееся по орбите вокруг другого тела, подчинено трём законам Кеплера. Нас будут интересовать только два из них - первый и третий.

Согласно первому закону Кеплера, тело, обращающееся вокруг Земли движется по эллипсу, в одном из фокусов которого находится центр Земли [2]. 


\section{Орбитальные элементы}

Для того, чтобы задать параметры и ориентацию орбиты околоземных объектов в пространстве, нужно указать 6 так называемых кеплеровских элементов (орбитальных элементов) (рис. 1). Орбита ИСЗ полностью задаётся шестью орбитальными элементами.

\begin{tabular}{|c|c|c|}
\hline & Наименование & $\begin{array}{l}\text { Обозначение } \\
\text { в программе }\end{array}$ \\
\hline 1. & $\begin{array}{l}\text { Большая полуось "а". Равна среднему расстоянию ИСЗ от центра } \\
\text { Земли. }\end{array}$ & D[ ].a1 \\
\hline 2. & Эксцентриситет "е" (см. формулу 1) - мера сплюснутости эллипса. & D[ ].a2 \\
\hline 3. & $\begin{array}{l}\text { Наклонение орбиты "i" к экваториальной плоскости Земли - угол } \\
\text { пересечения плоскости орбиты ИСЗ с плоскостью экватора Земли. }\end{array}$ & D[ ].a3 \\
\hline 4. & $\begin{array}{l}\text { Аргумент перигея (АП) } \omega \text { - угол, отсчитываемый в плоскости } \\
\text { орбиты ИСЗ от восходящего узла орбиты до точки перигея (точка, } \\
\text { где расстояние между ИСЗ и центром Земли наименьшее). }\end{array}$ & D[ ].a5 \\
\hline 5. & $\begin{array}{l}\text { Долгота восходящего узла (ДВУ) } \Omega \text { - угол, отсчитываемый в } \\
\text { плоскости земного экватора от восходящего узла до точки } \\
\text { весеннего равноденствия. Угол отсчитывается против часовой } \\
\text { стрелки, если смотреть с северного полюса мира. }\end{array}$ & D[ ].a4 \\
\hline 6. & $\begin{array}{l}\text { Средняя аномалия (СА) М0 - угол, отсчитываемый в плоскости } \\
\text { орбиты ИСЗ от перигея до ИСЗ на орбите. Угол отсчитывается } \\
\text { против часовой стрелки, если смотреть с северного полюса мира. }\end{array}$ & D[ ].a6 \\
\hline
\end{tabular}

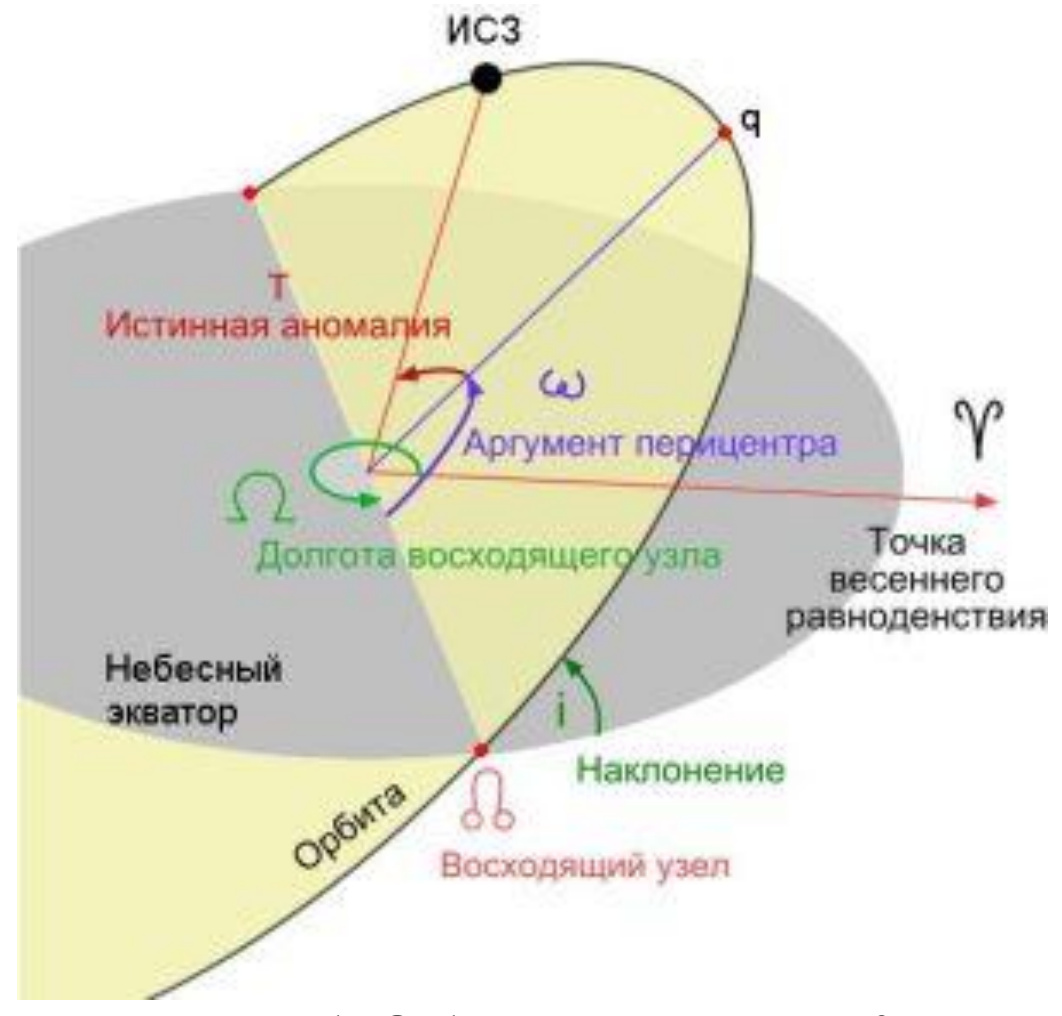

Рисунок 1 - Орбитальные элементы. [2]

Двигаясь по эллиптической орбите, объект ближе всего подлетает к центру Земли в перигее, а дальше всего находится в апогее. Также происходит - эволюция 
орбиты, прецессия орбиты, смещение восходящего узла за один виток [3], вращение эллиптической орбиты [1], атмосферное торможение, влияние давления света. В заключение нужно отметить, что перечисленные факторы влияния на эволюцию орбиты спутника не составляют полный список. Например, на ИСЗ воздействуют своим притяжением Солнце и Луна, но это воздействие в 10000 раз слабее действия экваториального "горба" Земли (Рис.2.), но его нужно учитывать для орбит с большим эксцентриситетом. Экваториальный "горб" также вызывает незначительные колебания плоскости орбиты ИСЗ при пересечении экваториальной плоскости. Наконец, неравномерность распределения масс под поверхностью Земли также сказывается на движении спутника.

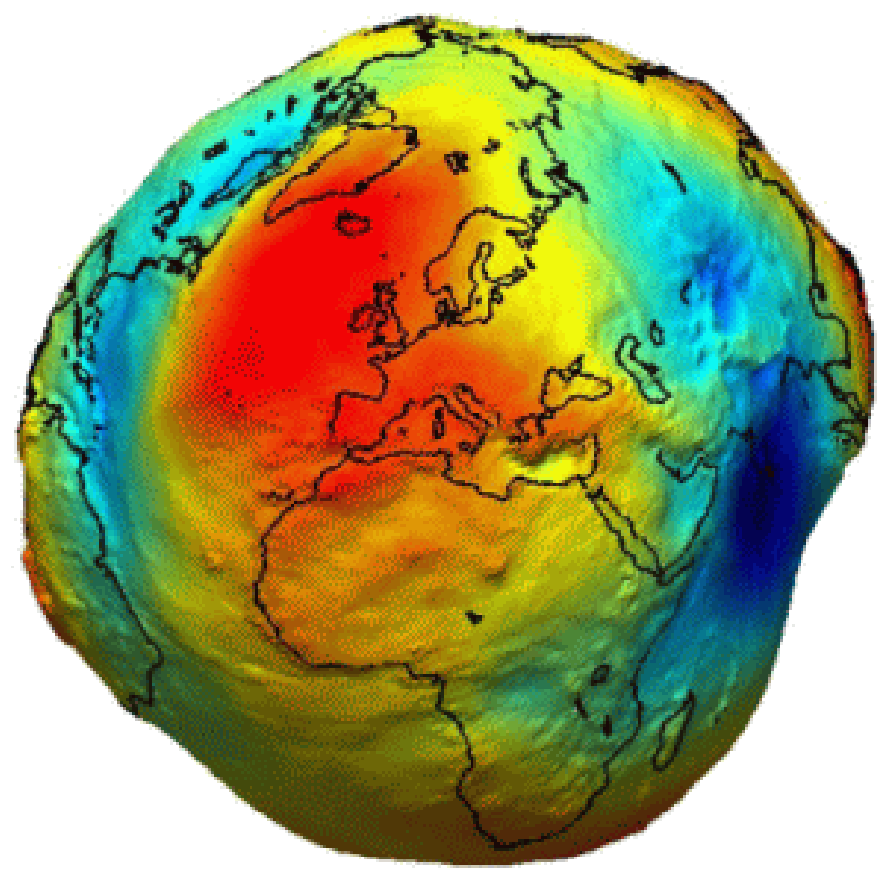

Рисунок 2 - Форма земного геоцда по данным ИС3 "GOCE" [4-5].

Используя все выше описанные алгоритмы разработаем интерактивную компьютерную математическую модель.

\section{База данных орбит объектов.}

Разработку будем вести посредством Directx11 и OpenGL в среде XE4. В качестве нормы примем среднюю длину радиуса Земли. Загрузим Quad объект шара и наложим текстуру. Траектории орбит будем загружать из базы данных. Все данные в базе - согласно таблице, указанных выше, орбитальных элементов:

Название орбитального объекта

Большая полуось

Эксцентриситет

Наклонение орбиты

Долгота восходящего узла

Аргумент перигея

Средняя аномалия
$\mathrm{D}[\mathrm{]}$.name

$\mathrm{D}[\mathrm{]}] \mathrm{a} 1$

$\mathrm{D}[\mathrm{]} \cdot \mathrm{a} 2$

$\mathrm{D}[\mathrm{a}] . \mathrm{a} 3$

$\mathrm{D}[\mathrm{]}] \mathrm{a} 4$

$\mathrm{D}[\mathrm{]}] . \mathrm{a} 5$

$\mathrm{D}[\mathrm{]} . \mathrm{a} 6$

Вращение модели в пространстве будем осуществлять в автоматическом режиме и вручную с помощью правой кнопки мыши в любую произвольную сторону. 
Увеличение и уменьшение размера с помощью колесика. Модель поддерживает более 100 млн. отдельных объектов, причем базу можно редактировать в реальном времени, а также при необходимости дополнить более подробными характеристиками и параметрами отдельных объектов, или связать с другими базами данных.

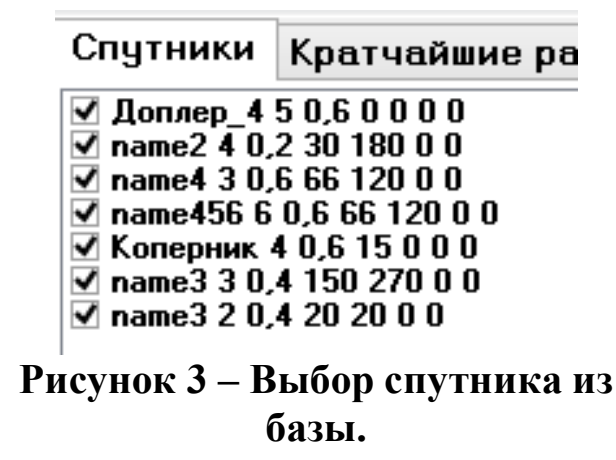

\begin{tabular}{|l|l|}
\hline Название спутника & name456 \\
\hline Большая полуось[а1] & 6 \\
\hline Эксцентриситет[а2] & 0,6 \\
\hline Наклонение орбиты[а3] & 66 \\
\hline Долгота восходящего узла[а4] & 120 \\
\hline Аргумент перигея(а5) & 0 \\
\hline Средняя аномалия(а6) & 0 \\
\hline
\end{tabular}

Рисунок 4 - Редактирование базы спутников.

Расчет кратчайших возможных расстояний между отдельными траекториями будем расчитывать как функцию минимизации длинны множества векторов соответствующих точек орбит в глобальной системе координат.

После нахождения, данные сортируются по возрастанию и выводятся на экран в указанием названий спутников. Имеется возможность не только найти минимальное расстояние но и определить глобальные координаты в каждом из случаев.

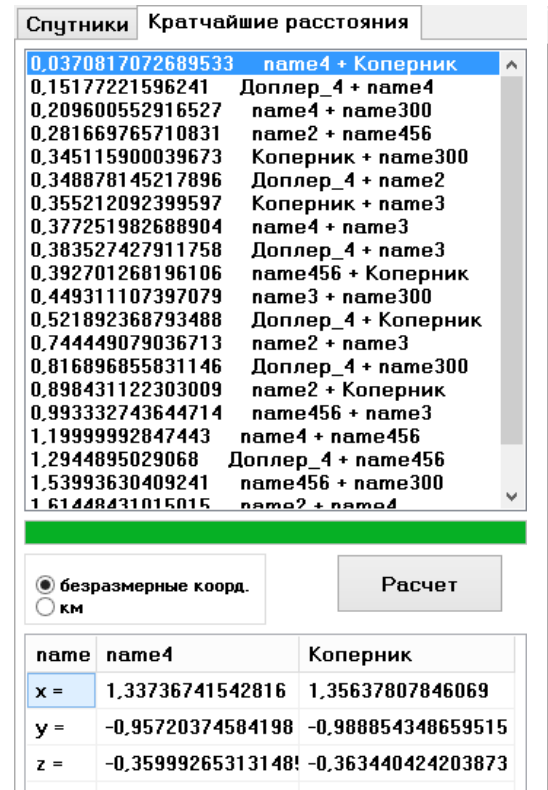

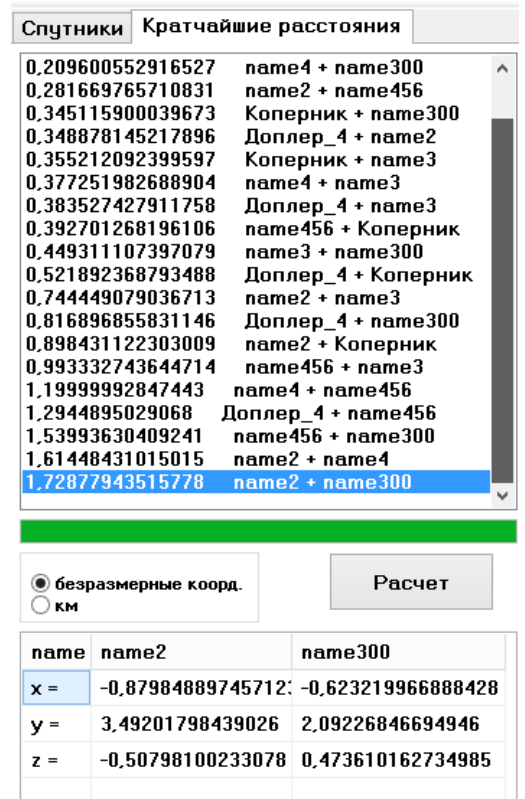

Рисунок 5 - Расчет кратчайшего, произвольного, и максимального расстояния между орбитами спутников в безразмерных координатах. 
ISPC «Economy, technology, education and prospects for 2014», 30.01.2014

\begin{tabular}{|c|c|c|c|}
\hline чики & \multicolumn{3}{|c|}{ Кратчайшие расстояния } \\
\hline \multicolumn{2}{|c|}{236.258746705371} & \multicolumn{2}{|c|}{ name4 + Коперник } \\
\hline \multicolumn{2}{|c|}{$\begin{array}{l}2403,58622044406 \\
2443,56897562349 \\
2502,01828035305 \\
2862,69664836369 \\
3325,13376668288 \\
4743,1097258685 \\
5204,69637350849 \\
5724,17578880262 \\
6328,82265567628 \\
7645,56165366399 \\
8247,58324533992 \\
9811,39888118324 \\
1026 \text { зББ72з20ก? } \\
\end{array}$} & \multicolumn{2}{|c|}{$\begin{array}{l}\text { Доплер_4 + name4 } \\
\text { name4 + name300 } \\
\text { name2 + name456 } \\
\text { Коперник + name300 } \\
\text { Доплер_4 + name2 } \\
\text { Коперник + name3 } \\
\text { name4 + name3 } \\
\text { Доплер_4 + name3 } \\
\text { name456 + Konepник } \\
\text { name3 + name300 } \\
\text { Доплер_4 + Konepник } \\
\text { name2 + name3 } \\
\text { Дonлep_4 + name300 } \\
\text { name2 + Konepник } \\
\text { name456 + name3 } \\
\text { name4 + name456 } \\
\text { Дonлep_4 + name456 } \\
\text { name456 + name300 } \\
\text { nama? + nama4 }\end{array}$} \\
\hline \multicolumn{3}{|c|}{$\begin{array}{l}\text { безразмерные коора. } \\
\text { км }\end{array}$} & Расчет \\
\hline name & name4 & & Коперник \\
\hline$x=$ & 1,33736741 & 2816 & 1,35637807846069 \\
\hline$y=$ & $-0,95720374$ & 1584198 & $-0,988854348659515$ \\
\hline$z=$ & $-0,35999265$ & 313148 ! & ! -0,363440424203873 \\
\hline
\end{tabular}

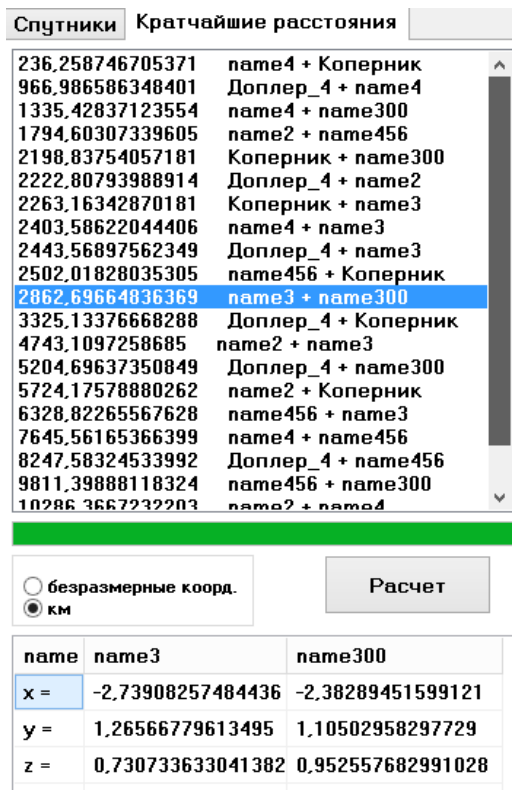

\begin{tabular}{|c|c|c|c|}
\hline \multicolumn{4}{|c|}{ Спутники Кратчайшие расстояния } \\
\hline \begin{tabular}{|l|}
1335,4 \\
1794,6 \\
2198,8 \\
2222,8 \\
2263,1 \\
2403,5 \\
2443,5 \\
2502,0 \\
2862,6 \\
3325,1 \\
4743,1 \\
5204,6 \\
5724,1 \\
6328.8 \\
7645.5 \\
8247,5 \\
9811,3 \\
10286, \\
1901.
\end{tabular} & $\begin{array}{l}2837123554 \\
0307339605 \\
3754057181 \\
0793988914 \\
6342870181 \\
8622044406 \\
6897562349 \\
1828035305 \\
9664836369 \\
3376668288 \\
097258685 \\
9637350849 \\
7578880262 \\
2265567628 \\
6165366399 \\
8324533992 \\
9828118324 \\
3667232203\end{array}$ & $\begin{array}{c}\text { name } \\
\text { name } \\
\text { Kone } \\
\text { Дonл } \\
\text { Kone } \\
\text { name } \\
\text { Дonл } \\
\text { name } \\
\text { name } \\
\text { Дonת } \\
\text { name2 } \\
\text { Дonл } \\
\text { name } \\
\text { name } \\
\text { name } \\
\text { Дons } \\
\text { name } \\
\text { name }\end{array}$ & $\begin{array}{l}4+\text { name300 } \\
2+\text { name456 } \\
\text { рник + name300 } \\
\text { ер_4 + name2 } \\
\text { pник + name3 } \\
4+\text { name3 } \\
\text { ер_4 + name3 } \\
456+\text { Konepник } \\
3+\text { name300 } \\
\text { ep_4 + Konepник } \\
+ \text { name3 } \\
\text { ep_4+name300 } \\
2+\text { Konepник } \\
456+\text { name3 } \\
4+\text { name456 } \\
\text { ep_4 + name456 } \\
456+\text { name300 } \\
2+\text { name4 }\end{array}$ \\
\hline \multicolumn{4}{|c|}{11014,5754540908} \\
\hline \multicolumn{3}{|c|}{$\begin{array}{l}\text { безразмерные коорд. } \\
\text { км }\end{array}$} & Расчет \\
\hline name & ame2 & & name30u \\
\hline$x=$ & $-0,8798488$ & $45 / 12$. & -0.623219966888428 \\
\hline$y=$ & 3.49201798 & 439026 & 2,09226846694946 \\
\hline$z=$ & $-0,5079810$ & 33078 & 0,473610162734985 \\
\hline
\end{tabular}

\section{Рисунок 6 - Расчет кратчайшего, произвольного, и максимального расстояния между орбитами спутников в системе СИ.}

Полученная интерактивная модель отображена на рисунках 7-10.

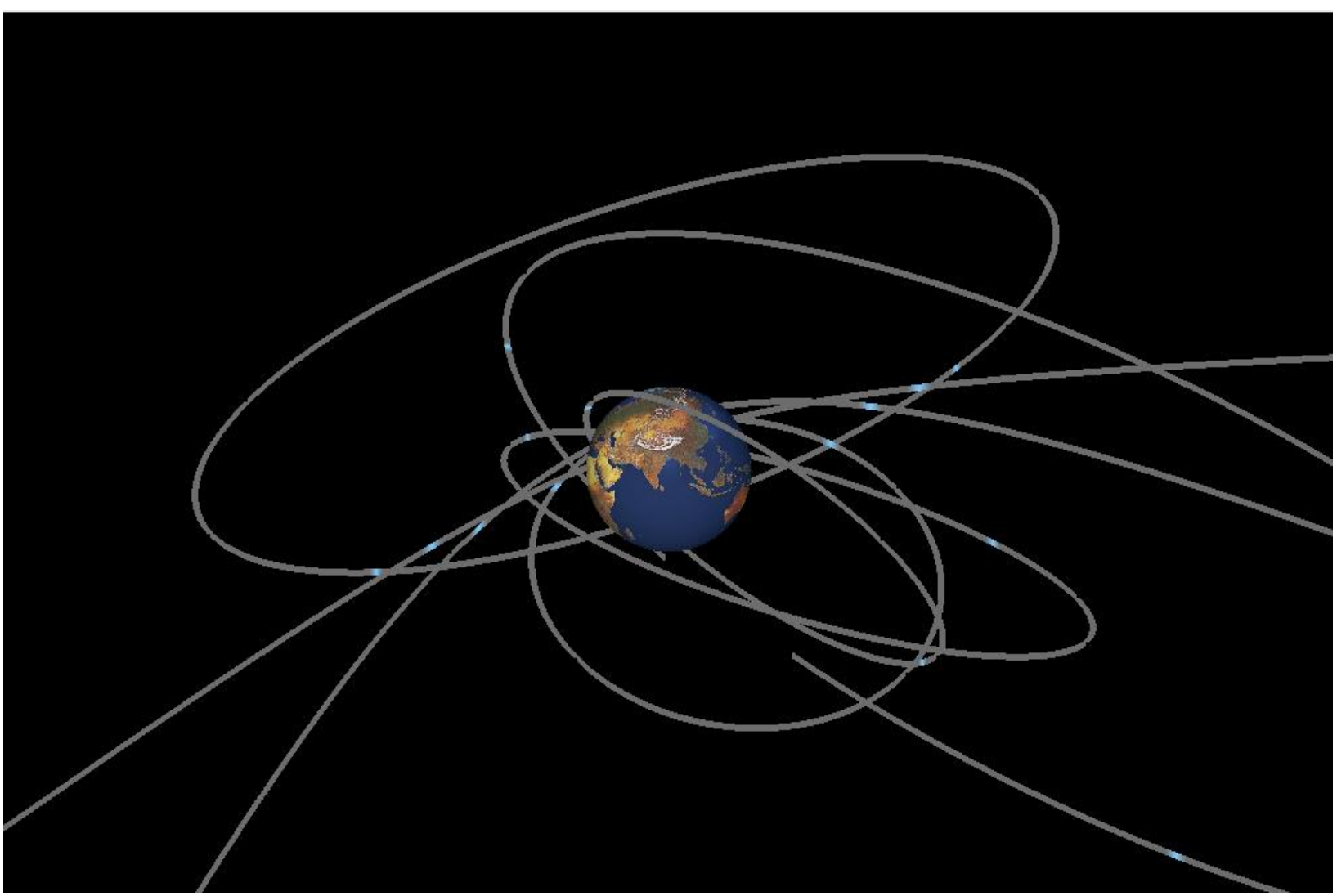

Рисунок 7 - Разработанная модель. 


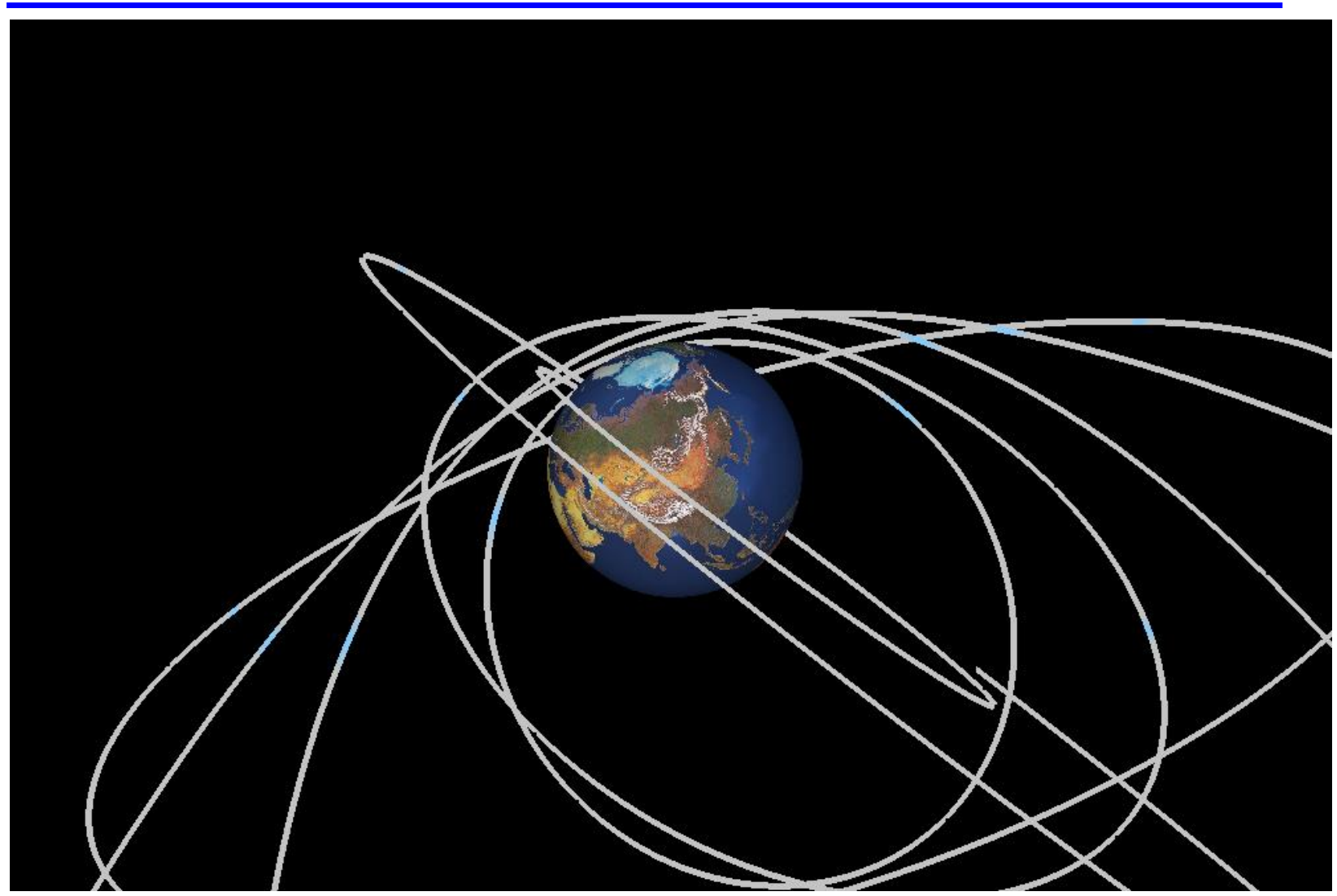

Рисунок 8 - Разработанная модель.

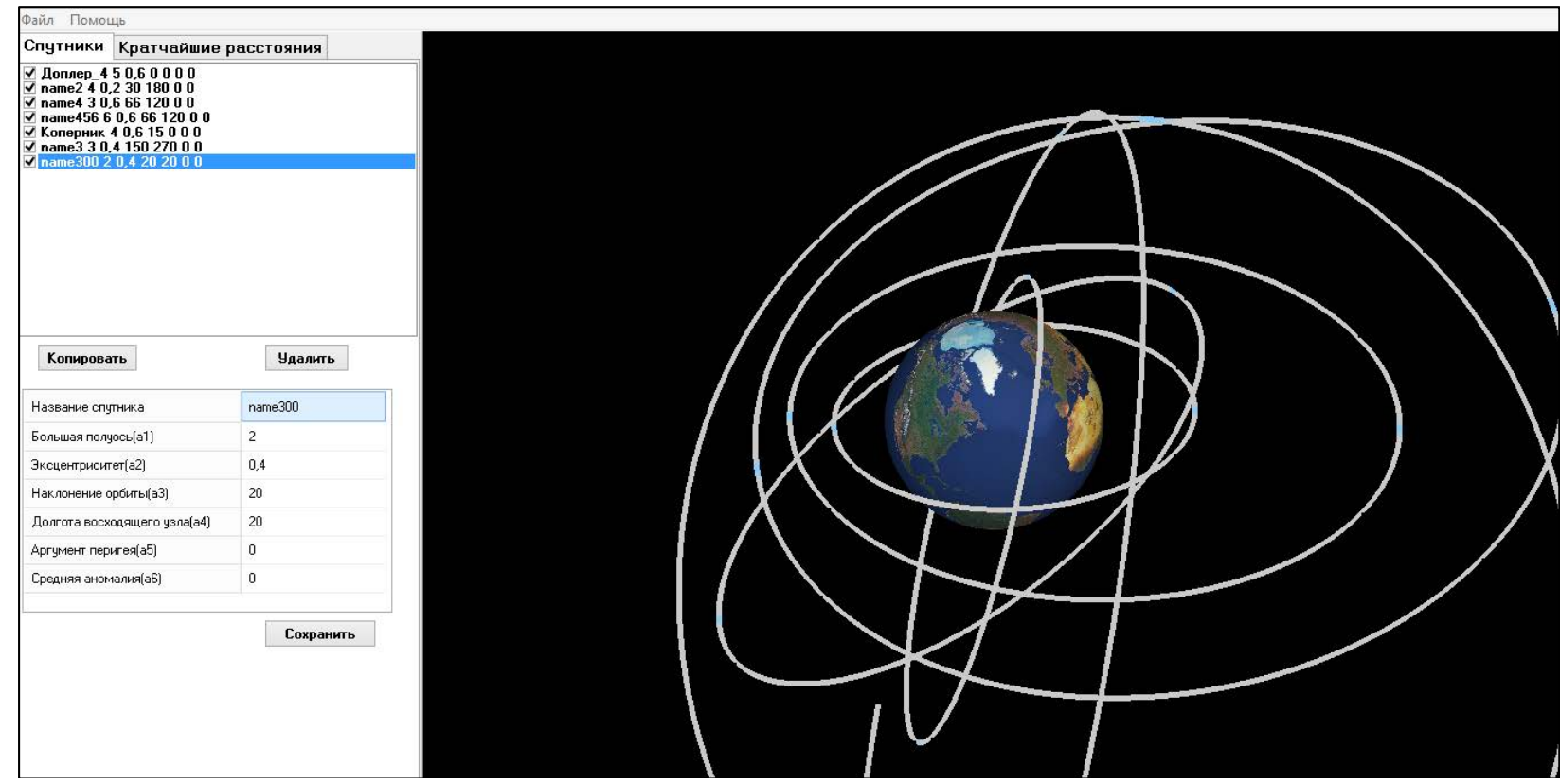

Рисунок 9 - Разработанная модель. 


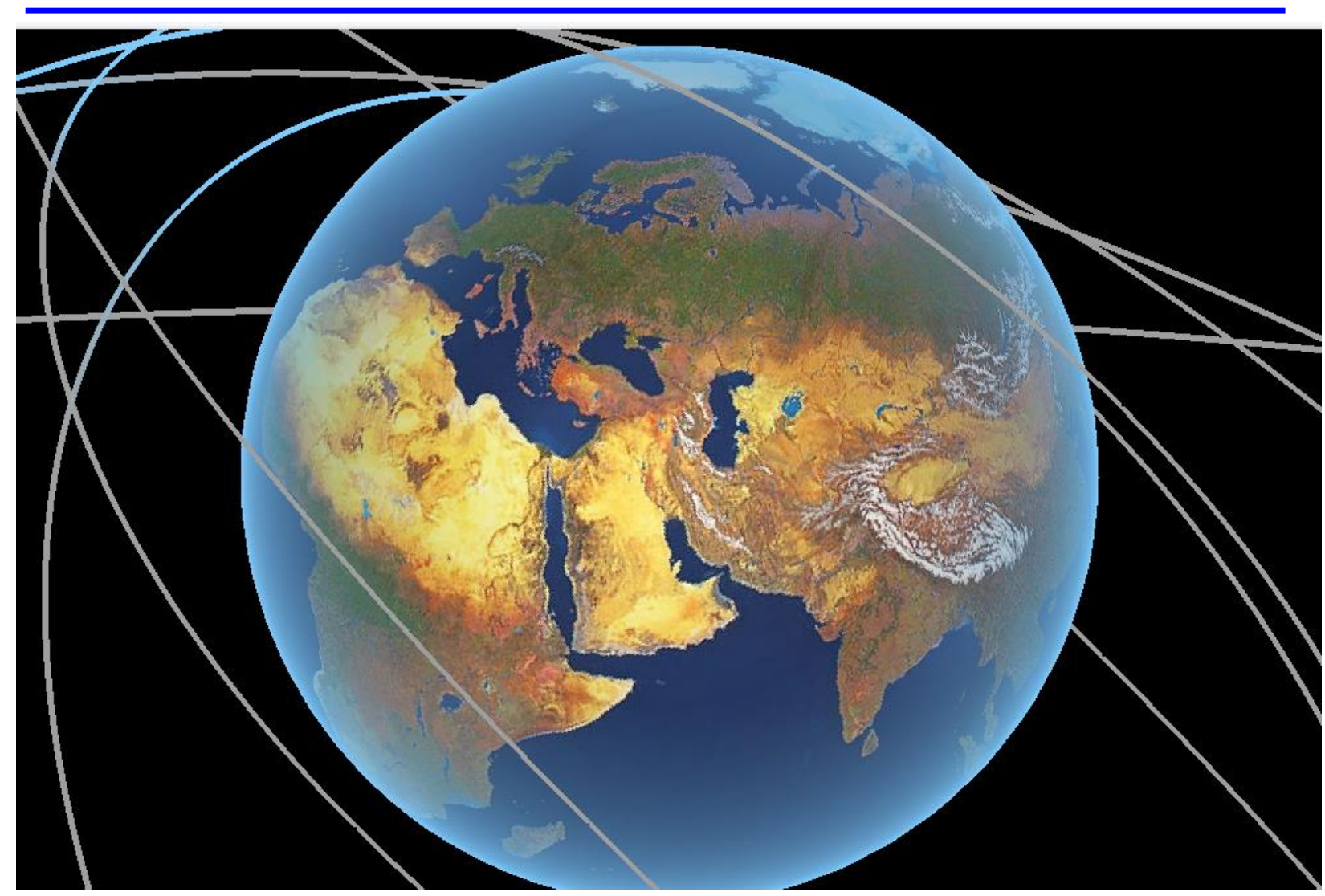

Рисунок 10 - Разработанная модель.

Полученная модель позволяет просчитывать минимальные расстояния между орбитами спутников и вероятности их столкновений. Модель основана на аппробированных методиках и алгоритмах расчета. Траектории спутников можно отображать и исследовать в реальном времени на данной интерактивной модели.

\section{ЗАКЛЮЧЕНИЕ}

В результате проведенного исследования и компьютерного моделирования получены следующие результаты:

- Разработана интерактивная компьютерная модель отображающая траектории околоземных объектов,

- Алгоритмы расчета траекторий движения спутников в глобальной системе координат связанной с Землей,

- Компьютерные алгоритмы трехмерных преобразований проекций,

- Алгоритмы определяющие все возможные минимальные расстояния между траекториями отдельных объектов.

Разработанные модели и программное обеспечение могут быть использованны как при научных исследованиях и построении более сложных систем слежения, так и в процессе преподавания.

\section{ЛИТЕРАТУРА}

1. Д. Кинг-Хили, "Искусственные спутники и научные исследования", М., 1963 г.

2. Кеплеровы элементы орбиты. [Электронный pecypc]. URL: http://ru.wikipedia.org/wiki/\%D0\%9A\%D0\%B5\%D0\%BF\%D0\%BB\%D0\%B5\%D1\%8 0\%D0\%BE\%D0\%B2\%D1\%8B \%D1\%8D\%D0\%BB\%D0\%B5\%D0\%BC\%D0\%B5\%D 
0\%BD\%D1\%82\%D1\%8B \%D0\%BE\%D1\%80\%D0\%B1\%D0\%B8\%D1\%82\%D1\%8B

3. Левантовский В.И., "Механика космического полёта в элементарном изложении", М., 1980 г.

4. Предложен новый метод определения формы Земли. [Электронный ресурc]. URL: http://mestechko.info/science/11010-predlozhen-novyj-metod-opredeleniya-formyzemli.html (дата обращения: 20.01.2014).

5. ESA показало самую точную "математическую форму Земли". [Электронный ресурс]. URL: HTTP:/LENTA.RU/NEWS/2011/04/01/GOCE (дата обращения: 12.01.2014).

6. Наблюдение искусственных спутников Земли. [Электронный ресурс]. URL: http://www.sat.belastro.net/glava1/glava1.php BelAstro.Net, Lupus, 01.09.2013 (дата обращения: 29.01.2014).

7. Наблюдение искусственных спутников Земли. [Электронный ресурc]. URL: http://www.sat.belastro.net/glava2/glava2.php BelAstro.Net, Lupus, 01.09.2013 (дата обращения: 29.01.2014).

ПРИЛОЖЕНИЕ

(алгоритмы и программа)

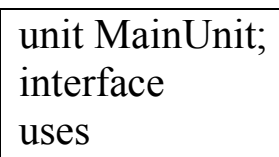


Dlina $=$ record

name1: string; name2: string; d:GLFloat; $\mathrm{x} 1$ :GLFloat; $\mathrm{y1}$ :GLFloat; $\mathrm{z1}$ :GLFloat; x2:GLFloat; y2:GLFloat; z2:GLFloat;

end;

TMainForm $=$ class $($ TForm $)$

Timer: TTimer;

Panel1: TPanel;

Timer1: TTimer;

ApplicationEvents1: TApplicationEvents;

TrackBar1: TTrackBar;

Memo1: TMemo;

PageControl1: TPageControl;

TabSheet1: TTabSheet;

CheckListBox1: TCheckListBox;

StringGrid1: TStringGrid;

BitBtn1: TBitBtn; BitBtn2: TBitBtn; BitBtn3: TBitBtn;

TabSheet2: TTabSheet;

BitBtn4: TBitBtn;

Memo2: TMemo;

RadioGroup1: TRadioGroup;

ProgressBar1: TProgressBar;

StringGrid2: TStringGrid;

ListBox 1: TListBox;

MainMenu1: TMainMenu;

N1: TMenuItem; N2: TMenuItem; N3: TMenuItem; N4: TMenuItem;

N5: TMenuItem;

procedure FormPaint(Sender: TObject);

procedure FormCreate(Sender: TObject);

procedure FormResize(Sender: TObject);

procedure TimerTimer(Sender: TObject);

procedure FormDestroy(Sender: TObject);

procedure FormClose(Sender: TObject; var Action: TCloseAction);

procedure FormMouseDown(Sender: TObject; Button: TMouseButton; Shift: TShiftState;

$\mathrm{X}, \mathrm{Y}$ : Integer);

procedure FormMouseMove(Sender: TObject; Shift: TShiftState; X, Y: Integer);

procedure Timer1Timer(Sender: TObject);

procedure ApplicationEvents1Message(var Msg: tagMSG; var Handled: Boolean);

procedure TrackBar1Change(Sender: TObject);

procedure CheckListBox1Click(Sender: TObject);

procedure BitBtn1Click(Sender: TObject);

procedure BitBtn2Click(Sender: TObject);

procedure BitBtn3Click(Sender: TObject);

procedure BitBtn4Click(Sender: TObject);

procedure ListBox1Click(Sender: TObject);

procedure N2Click(Sender: TObject);

procedure N4Click(Sender: TObject);

procedure N5Click(Sender: TObject);

private

// Переменные для инициализации OpenGL 


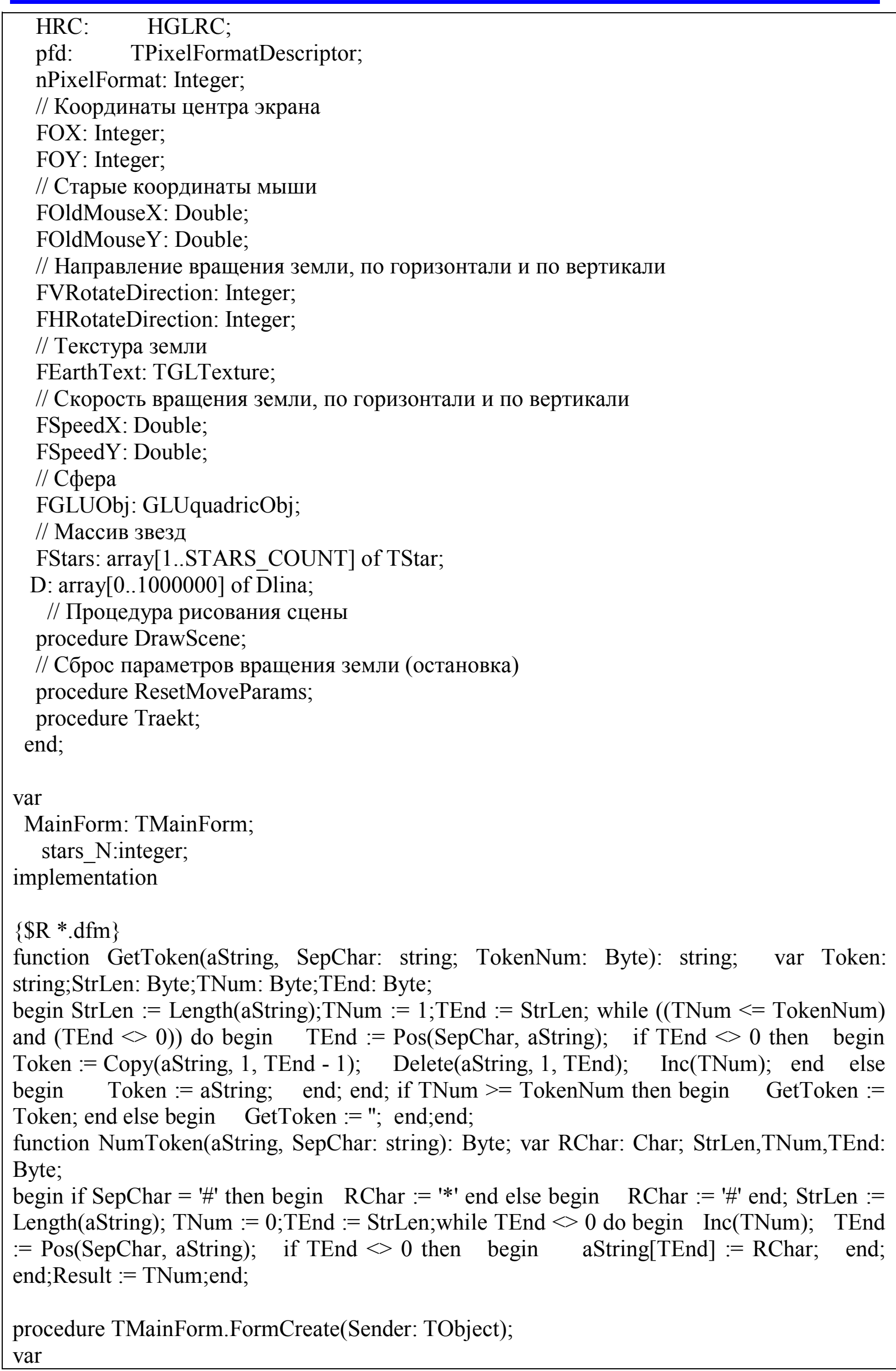




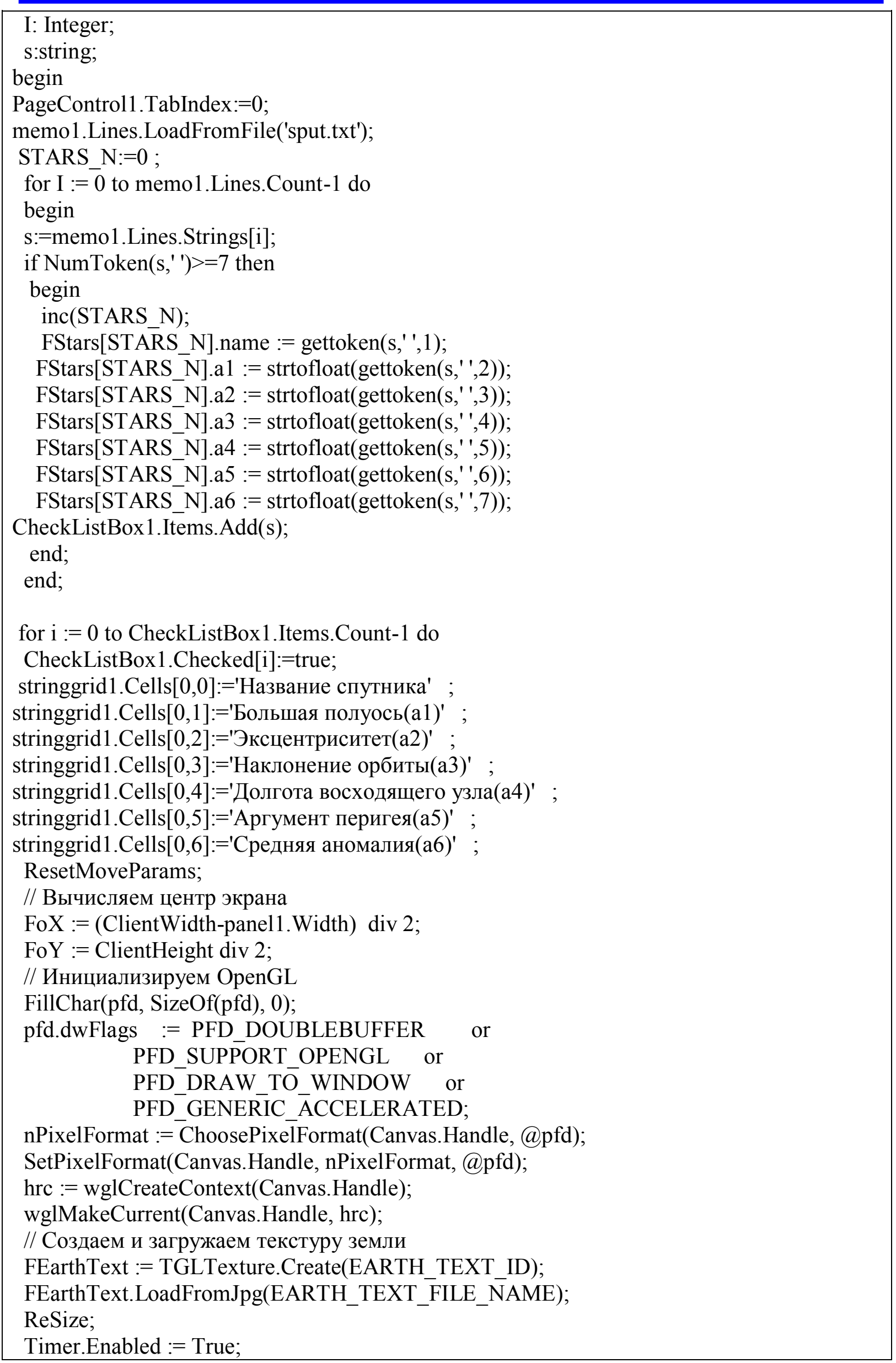




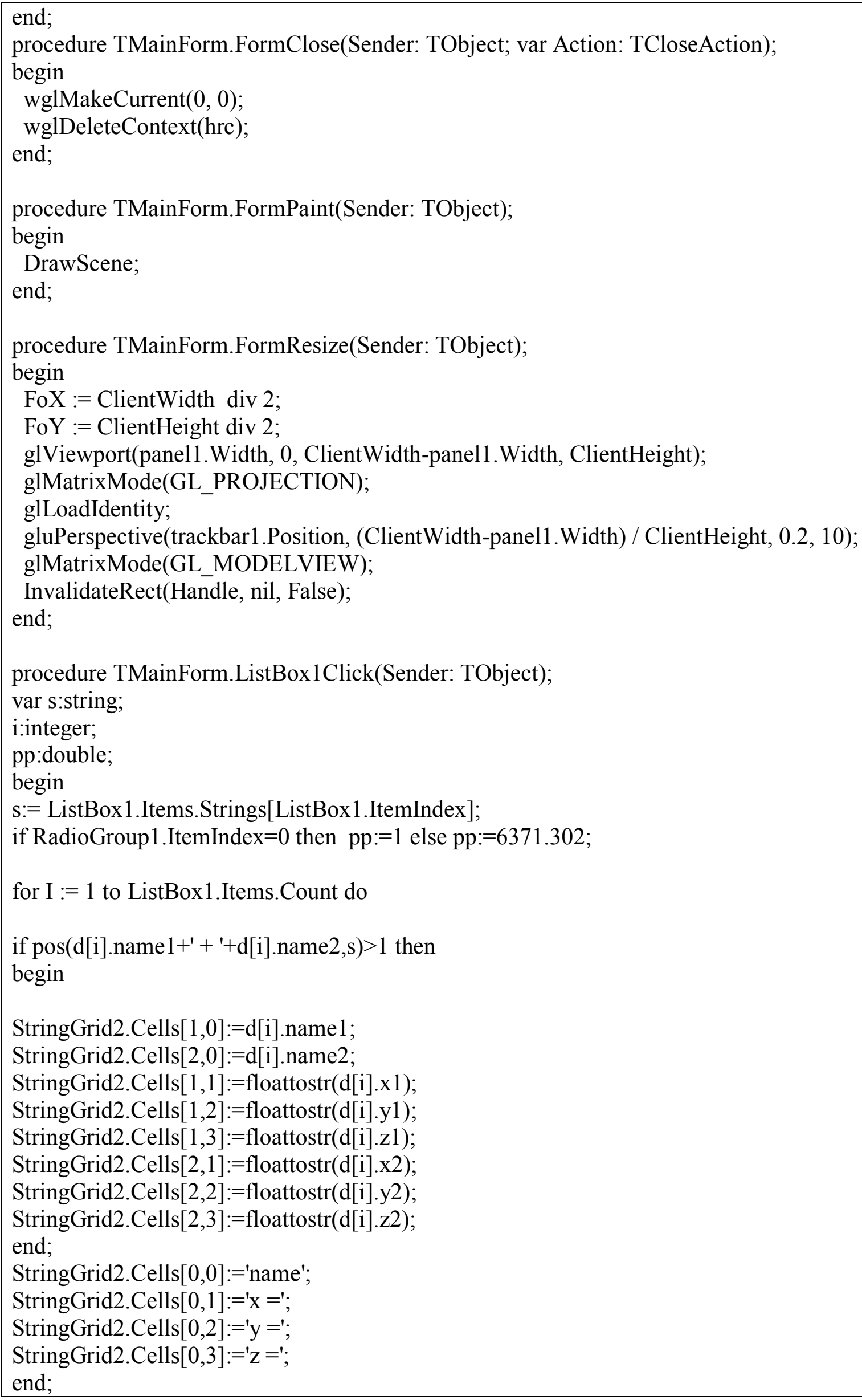


procedure TMainForm.N2Click(Sender: TObject);

begin

Application.Terminate;

end;

procedure TMainForm.N4Click(Sender: TObject);

begin

Showmessage('Математическое и компьютерное моделирование траекторий орбитальных объектов. Данная программа предназначенна для исследования траекторий движения околоземных объектов.');

end;

procedure TMainForm.Timer1Timer(Sender: TObject);

begin

application.ProcessMessages;

end;

procedure TMainForm.TimerTimer(Sender: TObject);

begin

DrawScene;

if FSpeedX $>0.2$ then

begin

FSpeedX := FSpeedX - 0.05;

end else

begin

FSpeedX $:=0.2$

end;

if FSpeedY $>0.05$ then

begin

FSpeedY $:=$ FSpeedY -0.05 ;

end else

begin

FSpeedY $:=0$;

end;

end;

procedure TMainForm.TrackBar1Change(Sender: TObject); begin MainForm.Resize; end;

procedure TMainForm.FormMouseDown(Sender: TObject; Button: TMouseButton; Shift: TShiftState; X, Y: Integer);

begin

FOldMouseX :=X; $\quad$ FOldMouseY $:=\mathrm{Y}$;

if ssLeft in Shift then ResetMoveParams;

end;

procedure TMainForm.FormMouseMove(Sender: TObject; Shift: TShiftState; X, Y: Integer); begin

if ssRight in Shift then 


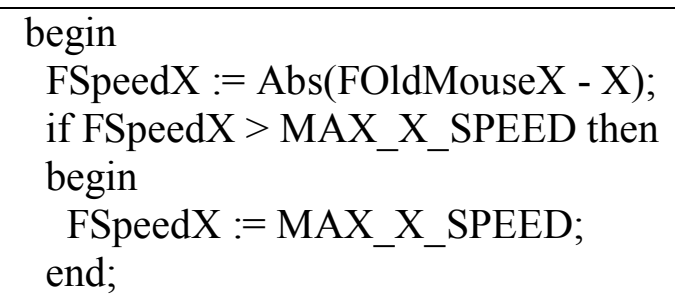




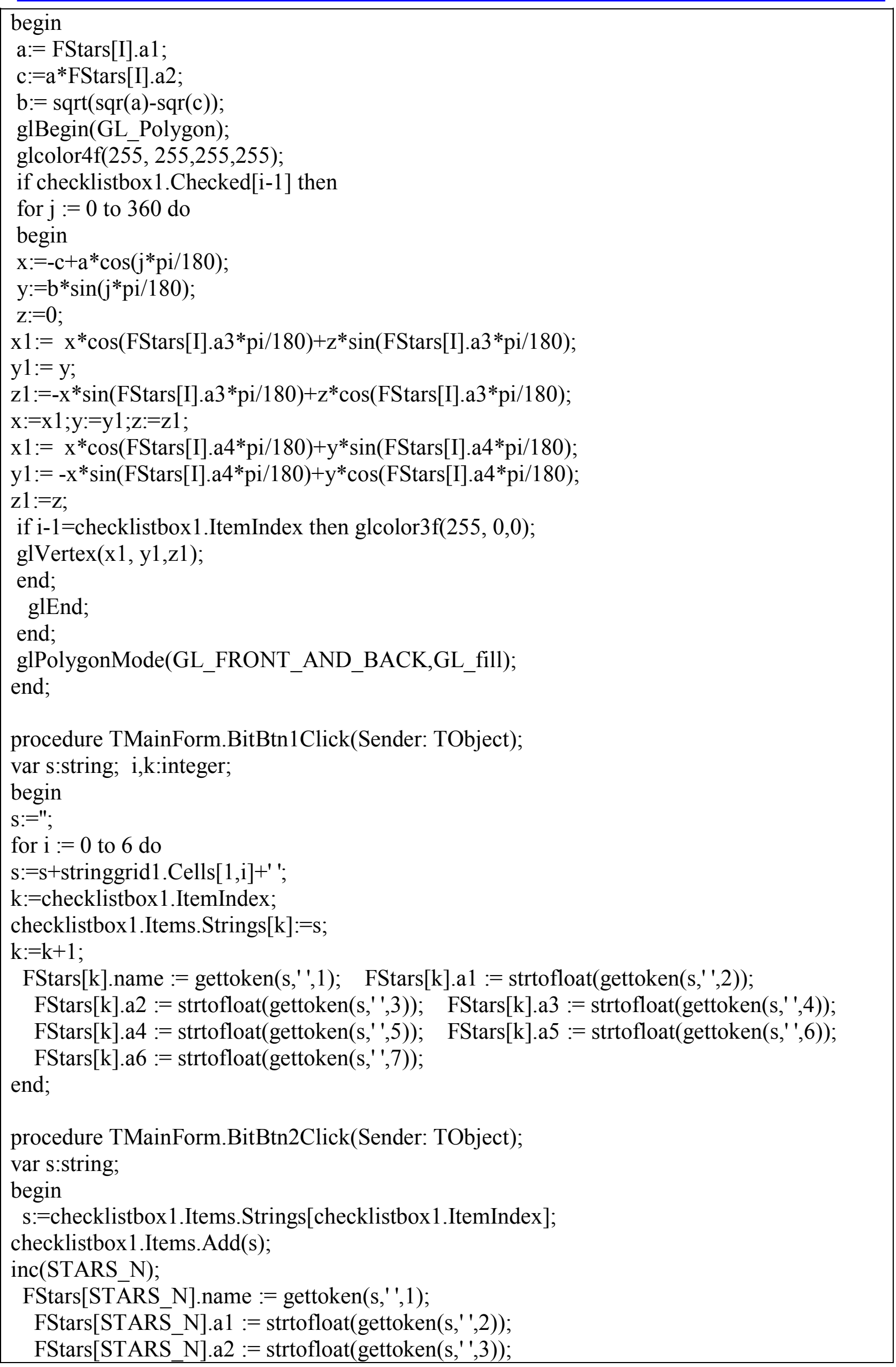




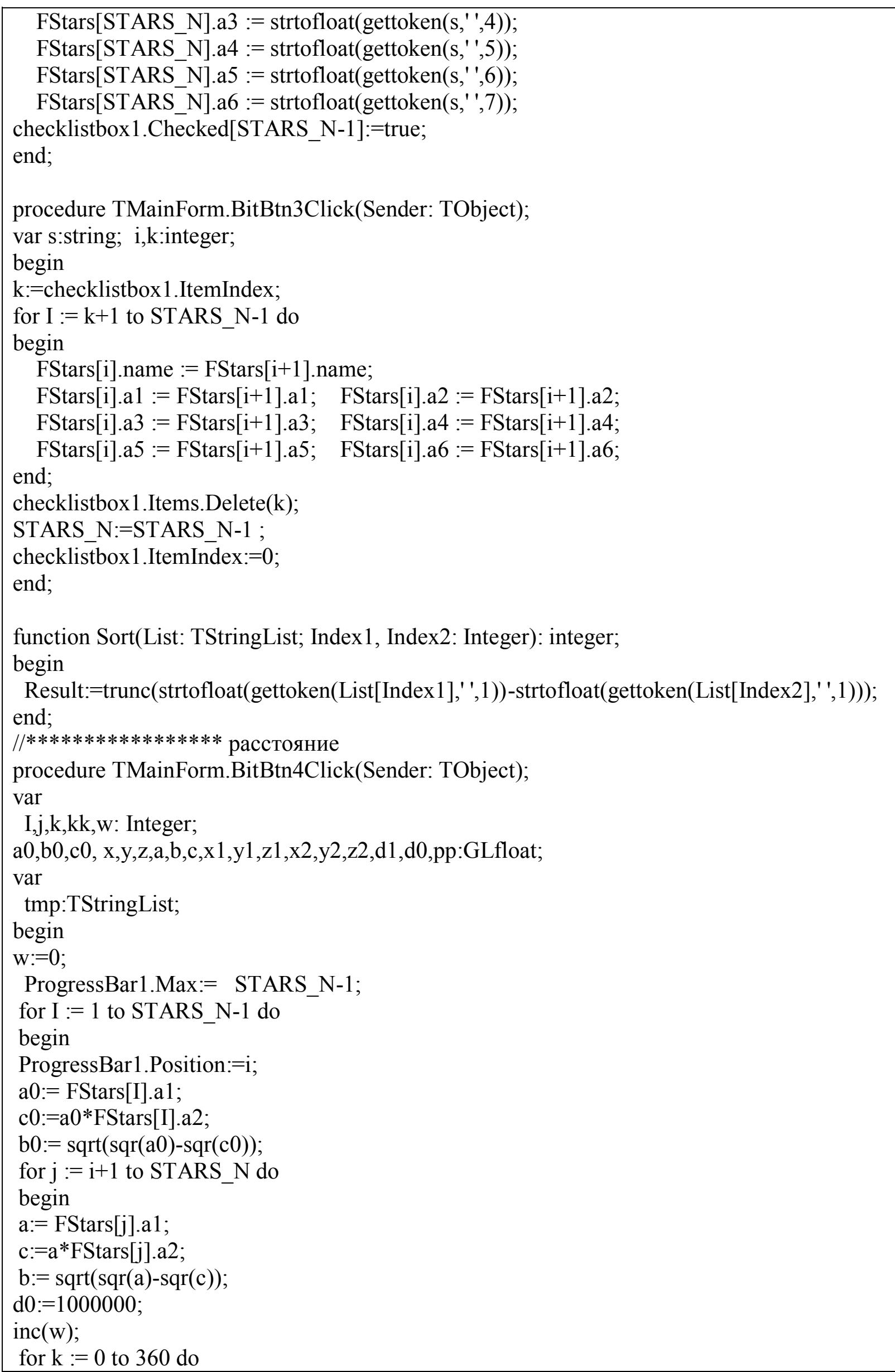




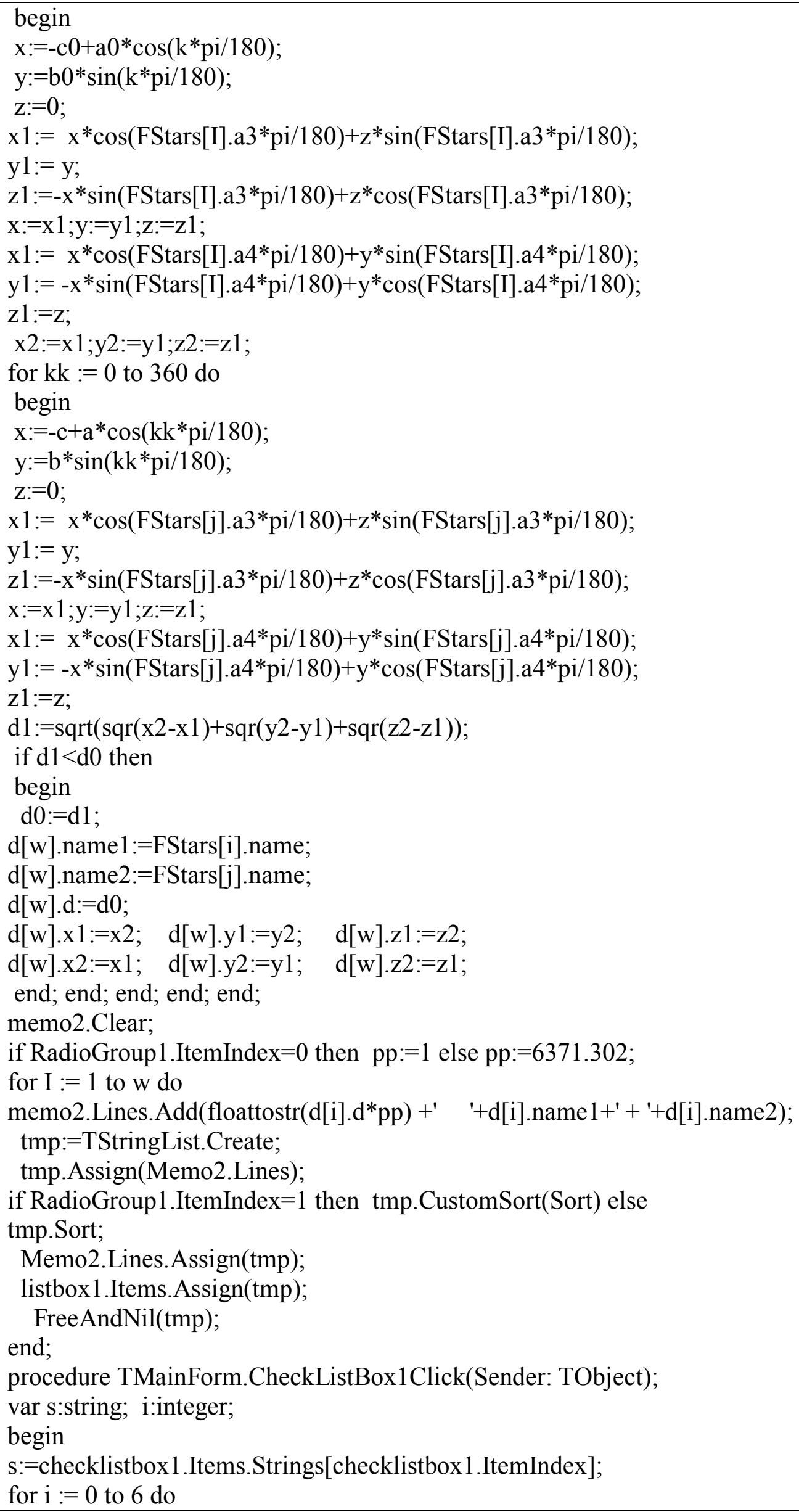




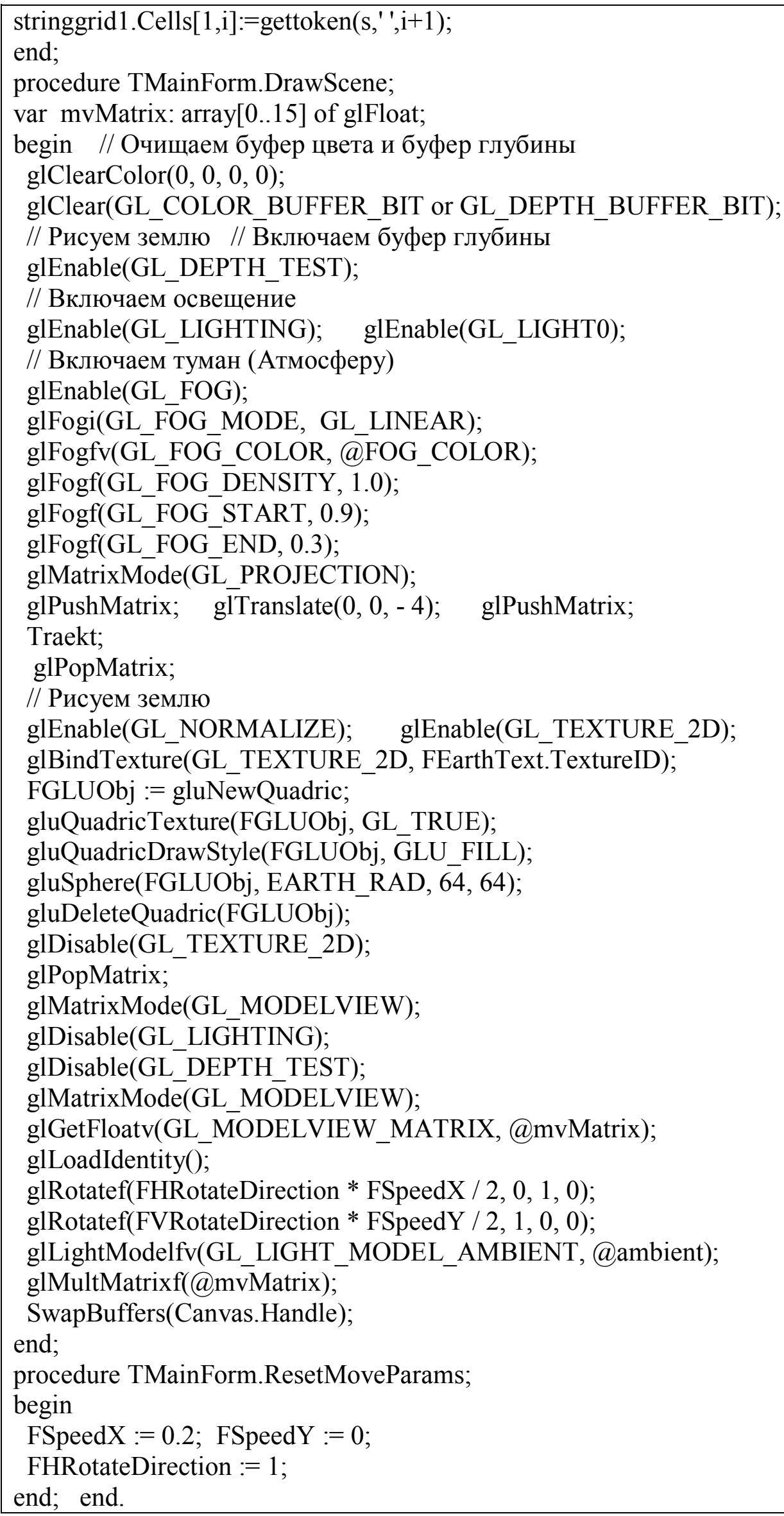

